\title{
Emprendimiento en Ecuador. Estudio de Caso: Provincia de Santa Elena
}

\section{Entrepreneurship in Ecuador. Case Study: Province of Santa Elena}

\author{
Valencia Cruzaty, Lilia \\ Universidad Estatal Península de Santa Elena \\ Santa Elena, 241702, Ecuador \\ lvalencia@upse.edu.ec
}

\begin{abstract}
Resumen
El emprendimiento es una de las soluciones fundamentales que se tratan de estimular en la provincia de Santa Elena, Ecuador. Para la implementación de las políticas de apoyo, es importante contar con conocimientos sistematizados acerca del mismo y así, evitar la improvisación en su pertinencia. El presente trabajo tiene como objetivo aportar un análisis que sirva de base a este empeño. Para ello además de consultas bibliográficas, se realizan análisis documentales propios del Ecuador y de la provincia de Santa Elena, que facilitan las valoraciones.
\end{abstract}

Palabras clave: Emprendimiento empresarial sostenible, Potencial innovador, Dimensiones del desarrollo sostenible. .

\begin{abstract}
Entrepreneurship is one of the fundamental solutions that try to stimulate in the province of Santa Elena, Ecuador. To implement support policies is important to have systematic knowledge about it to avoid improvisation in its relevance. This paper aims to provide a theoretical and epistemological system as a basis to this endeavor. This addition to bibliographic consultations, own documentary analysis of Ecuador and Santa Elena province, which facilitate the assessments are made.
\end{abstract}

Key words: sustainable entrepreneurship, innovative potential, dimensions of sustainable development..

\section{Introducción}

En Ecuador en el año 2015 se obtuvo un Índice de Actividad Emprendedora Temprana (TEA) de 33,6\%, por lo que aproximadamente 1 de cada 3 adultos había realizado gestiones para crear un negocio o poseía uno cuya antigüedad no superaba los 42 meses. (Ver Tabla No. 1) Esta cifra no ha presentado variaciones importantes en los últimos años. Lasio $\mathrm{V}$ et al, (2015)

De forma similar, el porcentaje de personas que poseía un negocio ya establecido (más de 42 meses) se mantuvo en el nivel de los últimos años $(17,4 \%)$. Siguiendo la tendencia, tanto los negocios emprendidos recientemente como los ya establecidos, se dedicaban en su mayoría al comercio (76\% y $51 \%$ respectivamente) y en lo fundamental orientados al mercado doméstico. La proporción de emprendimientos en comercio ha mantenido una tendencia creciente desde el 2010 (65\%).

En América Latina, Ecuador muestra de nuevo la TEA más alta, seguido por Chile $(25,9 \%)$ y Colombia $(22,7 \%)$.

En 2015, la TEA está compuesta por un $25,9 \%$ de negocios nacientes y un $9,8 \%$ de negocios nuevos. Es importante destacar que el $8 \%$ de los negocios nacientes fueron empezados por personas dueñas de un negocio nuevo y un $10 \%$ por dueños de negocios establecidos. Además, el 23,1\% de los adultos afirmaron haber emprendido aprovechando una oportunidad, mientras que el 10,3\% lo hicieron por necesidad. La tasa de emprendimiento por necesidad, al igual que en años anteriores, se encuentra por encima del promedio de América Latina y el Caribe.

En relación a los emprendedores por oportunidad, $26,6 \%$ afirmaron que lo hicieron para incrementar sus ingresos, un $28,8 \%$ lo hizo buscando independizarse y el $44,6 \%$ estuvo motivado también por necesidad, es decir, motivación mixta.

La TEA, tasa de emprendimientos tempranos en Ecuador se mantiene alta y sin cambios sustanciales en su composición: predominan los negocios nacientes, los negocios nuevos muestran tendencia decreciente; prevalecen los emprendimientos por oportunidad, pero el país aún posee una tasa alta de emprendimientos por necesidad, ubicándose por encima del promedio para economías de eficiencia, y aunque hay paridad de género en emprendimiento, más mu- 
jeres emprenden por necesidad. Sin embargo se observan, aunque incipientes, algunas transformaciones o tendencias positivas, que facilitarían abordar los desafíos que el país aún tiene en esta materia.

Los estudios de GEM (2015) llevan a insistir, en la necesidad de políticas de desarrollo productivo, integrales y articuladas, que incorporen al emprendimiento y la innovación. Del mismo modo se insiste en el papel de los emprendedores en el desarrollo empresarial y de los negocios establecidos en el ecosistema emprendedor; la salud de este no es solo responsabilidad de los gobiernos o la academia.

Hay que reconocer que tanto el Ecuador como la región poseen el "gen del comercio", por lo tanto es importante, de la mano con lo expresado antes, revalorizarlo y diseñar estrategias para hacerlo próspero y sostenible.

Tabla 1

Tasa de emprendimientos tempranos. Serie 2009- 2015

\begin{tabular}{cc} 
AÑO & TEA DE ECUADOR \\
\hline 2009 & $15,8 \%$ \\
2010 & $21,3 \%$ \\
2012 & $26,6 \%$ \\
2013 & $36,0 \%$ \\
2014 & $32,6 \%$ \\
2015 & $33,6 \%$ \\
\hline
\end{tabular}

Según Fonseca (2014), los sectores de mayor crecimiento son: la Administración pública y defensa, Planes de Seguridad Social de Afiliación, con un crecimiento 13,5\%; la Construcción con 9,6\%; los Servicios de Educación y Salud 7,61\%; la Intermediación financiera con 6,5\% y la Pesca con $5,74 \%$, el resto de actividades económicas han crecido por debajo del $5,01 \%$ de lo que creció la economía; el sector de menor crecimiento es la Agricultura, Ganadería, Caza y Silvicultura con 0,59\% y la Explotación de minas y canteras con $1,51 \%$.

Como se aprecia en las cifras expuestas se puede deducir que el sector público es quien ha sustentado el crecimiento de la economía ecuatoriana, de los últimos años, pues el petróleo ya se ha ido rezagando en este sentido con respecto al aporte del sector público.

No obstante estos logros, es notable la desaceleración económica que tiene lugar hoy en el Ecuador (Ver tabla No. 2) como consecuencia de la reducción del precio del petróleo desde fines de 2014, el difícil acceso a nuevas fuentes de financiamiento y la apreciación del dólar.

La PEA ${ }^{1}$, en septiembre de 2014, en el sector urbano, alcanzó 4,9 millones de ecuatorianos. De ellos, el $54 \%$ ocupado a pleno empleo, y en la categoría de subempleo se encuentra el 40,9\%, de los cuales existe un 4,4\% de trabajadores no remunerados. Estas cifras significan desafíos a las políticas socioeconómicas y por ello se incentiva

\footnotetext{
${ }^{1}$ Población Económicamente Activa
}

Tabla 2

Tasa de crecimiento real del PIB, en por ciento. Ecuador. (19992013)

\begin{tabular}{cc} 
Año & PIB \\
\hline 1999 & $-8,0 \%$ \\
2000 & $0,8 \%$ \\
2001 & $4,3 \%$ \\
2002 & $3,3 \%$ \\
2003 & $2,5 \%$ \\
2004 & $5,8 \%$ \\
2005 & $4,7 \%$ \\
2006 & $4,1 \%$ \\
2007 & $2,0 \%$ \\
2008 & $6,5 \%$ \\
2009 & $0,4 \%$ \\
2010 & $3,2 \%$ \\
\hline
\end{tabular}

el emprendimiento sostenible como una de las vías para mitigar y resolver de manera positiva estos indicadores.

Entre las mejoras experimentadas en el país, según el Banco Mundial (2015) se reconoce la reducción de la desigualdad, en tanto esta "ha sido más rápida que en la media de la región: el coeficiente de Gini se redujo de 54 a 46,7 entre 2006 y 2014, gracias a que el crecimiento benefició más a los más pobres". Estos progresos además de su valor cuantitativo encierran significados que en términos sociopsicológicos posibilitan el desarrollo de una mentalidad positiva hacia el protagonismo de los ciudadanos y ciudadanas de los sectores menos favorecidos en dirección a un emprendimiento exitoso.

En contraste con la etapa anterior a 2008, se plantea:

"esta nueva era de apoyo al emprendimiento ha sido movilizada desde el gobierno central, por un equipo estratégico que ha dado sostenibilidad al programa paraguas Emprendecuador. Como se puede ver en las estadísticas mostradas en este estudio, un $69 \%$ de actores del sistema ven al gobierno central como un agente de apoyo al emprendedor versus un $81 \%$ de los actores que en 2007 percibía que no había ningún apoyo por parte del Estado. Y un $56 \%$ de los actores opina que en los dos últimos años se pueden apreciar las herramientas de apoyo al emprendedor". (Catapulta, 2012, pág. 18).

Según (GEM-Ecuador, 2014) un $67.5 \%$ de los emprendedores se encontraban en el área urbana del país, tres puntos porcentuales por debajo de lo observado el año anterior. Esta reducción se atribuye principalmente a una disminución (del $7 \%$ ) en la proporción de emprendedores con negocios nacientes localizados en el área urbana, que en 2014 alcanzó un $70 \%$. No obstante, esta supera a lo observado para emprendedores con negocios nuevos (61.9\%). A pesar de un ligero incremento registrado en esta tasa con respecto al año anterior, se observa entonces en general, una 
tendencia decreciente en el porcentaje de negocios nuevos que se encuentran en el área urbana. En contraste, se aprecia que para el año 2016 un $60 \%$ de los dueños de negocios establecidos se encuentran localizados en el área rural del país, situación que se ha mantenido desde el 2008.

Pero ese no es el caso de la provincia de Santa Elena, donde en el área rural, la dinámica del emprendimiento se percibe más deprimida. La provincia de Santa Elena, lugar donde se desarrollará el estudio es eminentemente rural, con muy poca área urbana. Dos de sus tres cantones son rurales. El Banco Nacional de Fomento y otras entidades bancarias, la Secretaría Nacional del Migrante y el Ministerio Coordinador de la Producción (MIPRO), mediante el Código de la Producción respalda la creación de empresas con incentivos. Entre ellos la exoneración del Impuesto a la Renta por cinco años a empresas que fabriquen bienes usualmente importados como abonos, fertilizantes, electrodomésticos, detergentes, cosméticos, calzado, entre otros. También hay deducciones en Impuesto a la Renta a quienes hagan capacitaciones para investigación, desarrollo e innovación tecnológica. Cada una de estas instituciones financia hasta un monto de US 60 mil dólares.

De otra parte, la sostenibilidad de los emprendimientos es uno de los desafíos que presenta el Ecuador. Por ello, es necesario estudiar y al mismo tiempo estimular la creación de pequeñas y medianas empresas y la economía familiar en negocios que amplían la plataforma de producción y consumo de diversas zonas del país, como sucede en la Provincia de Santa Elena.

Según el INEC, como resultado del censo del año 2010, se indica que en Santa Elena existe una población de 308.693 habitantes, con una población económicamente activa de 108.830 , de la cual 29.021 personas trabajan por cuenta propia.

Según dicho organismo la proyección de población en la Península de Santa Elena al 2015 corresponde a 358.896 y en el 2020 se calcula que hayan 401.178 personas, por lo que se observa que la población crece más rápido que los empleos existentes.

La poca oportunidad de obtener un empleo formal ha abierto la posibilidad de generar emprendimiento tanto en jóvenes como en adultos, de forma individual o grupal, oportunidad que ha permitido aprovechar la predisposición a desarrollarse a pesar de vivir en un mundo con economías limitadas, donde el empleo formal es escaso y el informal es difícil de mantener.

En la Provincia durante mucho tiempo prevaleció, y aún subsisten en una gran parte de la población, los criterios de que un emprendimiento es cualquier acto comercial que permita la subsistencia y sin importar las condiciones en que se desarrolla. Por lo que es usual encontrar en algunas áreas muchos puestos de ventas y de servicios que satisfacen necesidades perentorias de la población, los mismos que no cuentan con personería jurídica, ni identidad comunicacional intencionada en la mayoría de los casos. No se considera el emprendimiento como un proceso donde la inversión inicial puede llegar a reportar un mejoramiento de condiciones de vida. De lo que se desprende que el emprendimiento constituye un medio de subsistencia y no como generación de riqueza.

En el actual territorio de la provincia de Santa Elena se inicia la explotación de petróleo en el Ecuador a gran escala en 1911, con resultados productivos positivos. Por la actividad de extracción intensiva, los pozos de la localidad se fueron extinguiendo y hoy solo existen algunos cuya productividad es insignificante si se compara con los niveles alcanzados en décadas pasadas.

Aun así y en ese contexto, la rama de los hidrocarburos continúa siendo un área productiva importante, sus aportes a la economía se encuentran disminuidos por su dependencia del sector petrolero. Esta situación, unida a la apreciación del dólar ha afectado la balanza comercial del país, las inversiones y la competitividad de sus exportaciones.

En la actualidad, el emprendimiento cobra alto significado para los moradores de Santa Elena porque muchas personas tienen la necesidad de lograr su independencia y estabilidad económica. El desempleo y la deficiente calidad de los empleos que existen, han hecho que algunas personas generen sus propios recursos convirtiéndose de empleados en empleadores.

Desde el punto de vista del emprendedor, se comprende que es necesario tener una gran determinación para dejar la "estabilidad" económica que ofrece un empleo y arriesgarse como empresario emprendedor, teniendo en cuenta que este no siempre gana como lo hace un asalariado, que tiene asegurado mes tras mes un ingreso mínimo, mientras que el emprendedor naciente tiene que enfrentarse a múltiples dificultades y obstáculos en el proceso, por tanto se encuentran muchas veces en una situación de incertidumbre, y que si no están preparados de forma apropiada o no tienen el apoyo necesario del sector público y privado es probable que esos nuevos negocios tengan que cerrar y los emprendimientos pensados no sean viables.

Aún con los beneficios a nivel nacional como política de Estado, el Programa de Desarrollo y Ordenamiento Territorial de Santa Elena (PODT, 2011), refiere que:

“... existe poca inversión en el sector económico de la provincia, una de las razones para que esto suceda se debe a que el acceso al crédito para las Pequeñas y Medianas Empresas es limitado, ya que no existen las garantías necesarias para que las entidades financieras puedan recuperar el dinero entregado a través de créditos. A todos estos inconvenientes hay que sumarle los escasos recursos del presupuesto del Estado para el fomento productivo a nivel provincial, lo que limita el desarrollo de este sector".

En ese sentido, el Gobierno Autónomo Descentralizado de la Provincia Santa Elena ha ejecutado el programa de capacitación denominado "Inclusión Digital", cuyo objetivo es enseñar y formar a los adolescentes participantes a este curso en materias tecnológicas, ofreciendo conceptos 
teóricos y prácticos, incentivando así los emprendimientos particulares de futuros negocios. También cuenta con un proyecto que promueve la ayuda financiera reembolsable denominado "Fondo Semilla", con la finalidad de promover emprendimientos de las mujeres de la provincia para impulsar la mejora de la calidad de vida. Para marzo del 2015 contaba con cerca de mil mujeres emprendedoras distribuidas en los tres cantones provinciales.

Las actividades productivas principales a las que se dedican las beneficiarias son:

- Comercio (venta por catálogo)

- Pesca

- Artesanías

- Venta de abarrotes y artículos de bazar

- Venta de ropa

Se considera que la realidad económica de la provincia Santa Elena con las oportunidades de crecimiento $\mathrm{y}$ el entorno en el que se desenvuelve el emprendedor en el territorio, exige un flujo de información continua y actualizada que permita conocer y divulgar las áreas en las que incursionan los nuevos emprendedores, que hoy día no se cuenta con información actualizada al respecto, por lo que no es posible determinar si existe un enfoque adecuado hacia el sector donde hay más oportunidades de crecimiento o si sería recomendable redireccionar las áreas, hacia las labores de producción y servicios más convenientes.

En el desafío de mantener los niveles de reducción de la pobreza, la provincia de Santa Elena comienza a desarrollarse económica y productivamente a partir de la diversificación en la producción y los servicios, donde el emprendimiento ocupa un lugar importante tanto en áreas urbanas como rurales, aunque algunos de ellos no resultan viables y terminan por extinguirse en poco tiempo.

Según datos del MIPRO (2012) en la provincia Santa Elena, dadas sus características, existen posibilidades reales y potenciales para establecer emprendimientos en diferentes áreas de desarrollo (Ver Tabla no.3).

Se aprecia que la mayor actividad productiva y de negocios se asocia a la rama agropecuaria y a la ictiológica. Los de mayor potencial se vinculan a la diferenciación y diversificación productiva de la zona. De igual modo se valoran los productos agroindustriales destinados tanto para la agroexportación como para el consumo nacional, sobre todo aquellos de procesamiento de pescado y moluscos, y la industria de derivados como la harina de pescado.

Dentro de los servicios, se percibe un buen potencial para el desarrollo de turismo de intereses especiales, que va desde el turismo de observación, hasta el turismo de salud, comunitario, pasando por etnológico y de aventura, y turismo convencional de playa.

También aparecen en este territorio con alto potencial, las posibilidades de desarrollo de la industria de producción de biocombustibles alternativos, a partir del procesamiento de caña de azúcar para etanol, que dada la existencia de extensas superficies aptas para este cultivo, es posible integrarlo a proyectos de establecimiento de plantas de extracción, con potencial de generación termoeléctrica.

Tanto las áreas de desarrollo como los negocios reales y potenciales, suponen una manera de relación especial con el medio ambiente y con el desarrollo sostenible de la provincia. Los principales problemas que se identifican con el desarrollo sostenible en Santa Elena (GADP, 2012) según el "Plan de Desarrollo y Ordenamiento Territorial 2012-2021", que están relacionados con el medio ambiente son:

- Déficit hídrico y erosión de suelos

- La provincia es seca y carece de fuentes de agua propia y segura

- Salinización del suelo y agua del subsuelo

- Suelo contaminado

- Agua contaminada

- Aire contaminado

- Ambientes marinos y costeros contaminados

- Disminución de la fauna silvestre

En el ámbito de la economía popular y solidaria la prefectura (GADP, 2012) plantea problemas como:

- Presencia desordenada en principales centros urbanos, lo que contribuye al caos y agrava la falta de planificación y zonificación de los centros más poblados.

Tabla 3

Negocios actuales y potenciales de Santa Elena.

\begin{tabular}{|c|c|c|c|}
\hline No. & Áreas & Negocios & Tipo \\
\hline 1 & Agricultura y Agroindustria de exportación & $\begin{array}{l}\text { Comercialización de carnes rojas } \\
\text { Producción y Exportación de Frutas frescas exóticas y superfrutas: Pińa, Mango, Papaya. } \\
\text { Procesamiento y comercialización de carnes procesadas } \\
\text { Exportación Agroindustria (Jugos, Conservas, Snacks, deshidratados de frutas) incluye IQF, purés, néctares. }\end{array}$ & $\begin{array}{l}\mathrm{R} \\
\mathrm{R} \\
\mathrm{P} \\
\mathrm{R}\end{array}$ \\
\hline 2 & Agropecuarios de consumo nacional & $\begin{array}{l}\text { Producción y Exportación de maíz para balanceado en industrias de proteína animal } \\
\text { Procesamiento y comercialización Avicultura (Pollos, Patos). }\end{array}$ & $\begin{array}{l}\mathrm{R} \\
\mathrm{R}\end{array}$ \\
\hline 3 & Construcción y materias primas & Construcción de viviendas & $\mathrm{R}$ \\
\hline 4 & Turismo & Turismo de intereses especiales & $\mathrm{P}$ \\
\hline 5 & Productos de la pesca y acuacultura & $\begin{array}{l}\text { Exportación de pesca blanca fresca y congelada } \\
\text { Exportación de atún en conserva } \\
\text { Harina de Pescado } \\
\text { Camarón congelado } \\
\text { Maricultura de Peces }\end{array}$ & $\begin{array}{l}\mathrm{R} \\
\mathrm{R} \\
\mathrm{R} \\
\mathrm{R} \\
\mathrm{R}\end{array}$ \\
\hline 6 & Energías Alternativas & Producción de biocombustibles a partir de cańa para etanol & $\mathrm{P}$ \\
\hline
\end{tabular}


- Bajo nivel de asociatividad. Esta es una característica en las distintas modalidades de autoempleo y con bajos de nivel de calificación, situación que restringe su capacidad para presentar alternativas de reubicación en el caso de comerciantes ambulantes o de puesto fijo urbanos.

- Disciplina y cumplimiento laboral, que radica básicamente en el incumplimiento de compromisos para la elaboración y/o reparación de bienes, situación que lejos de ser una situación aislada, afecta a los gremios en su conjunto, a la autoimagen y proyección del sector.

\section{CONCLUSIONES}

Entre los principales elementos de análisis para el estudio del emprendimiento en la provincia de Santa Elena resulta imprescindible el desarrollo y la articulación de proyectos dirigidos a la sostenibilidad en todas sus variantes y ámbitos. Al mismo tiempo impulsar el desarrollo del potencial tecnológico mediante estudios que demuestren su factibilidad en el territorio.

Esta investigación considera como condición sine qua non que solo el emprendimiento que articula de modo permanente las dimensiones económica, social, ambiental, institucional, cultural y de la personalidad, aporta de forma predeterminada al desarrollo sostenible del territorio en que se encuentra.

\section{Referencias Bibliográficas}

Acs, A. E. y. S. L., Z. J. (2004). "national systems of entrepreneurship: measurement issues and policy implications.research policy.

Aldrich, H. (2005). Entrepreneurship. en neil j. smelser and richard swedberg (eds.). the handbook of economic sociology.

Altran. (2015). Índice de innovación 2015. análisis de la capacidad innovadora. Descargado de http: / / www.altran.es/innovacion/ indice-altran/indice-de-innovacion . html\#. VwwYbnry3 IU

Arboleda, O. L. y. Z. H. (2011, enero-junio de 2011). Condiciones clave para el éxito y sostenibilidad de los emprendimientos solidarios de medellín., 14(28), 77-94.

Arendt, H. (2002). La condición humana.

Arias Ramírez, L. V. M. L. A. R. O., R. Sánchez Hernández. (2015). Criterios para la identificación y definición de territorios con potencial de desarrollo productivo para el establecimiento de zonas económicas especiales de desarrollo (zeed) en costa rica. , 33(1), 89-119.

Barcellos de Paula, L. (2010). Modelos de gestión aplicados a la sostenibilidad empresarial. (Doctor en Empresas).

Brundtland, G. H. (1987). Comisión mundial para el medio ambiente y el desarrollo de la onu. [Informe].
C., H. (2012). Marco institucional para el desarrollo sostenible: el mayor desafío de la cumbre río + 20. Descargado de http://www. fesenergiayclima . org

Cantillon, R. (2010). An essay on economic theory.

Cardona M, T. J., Vera L. (2008). Las dimensiones del emprendimiento empresarial: la experiencia de los programas cultura y fondo emprender en medellin.

Castillo, A. (1999). Estado del arte en la enseñanza del emprendimiento. programa emprendedores como creadores de riqueza y desarrollo regional intec.

Catapulta. (2012). Cimientos de emprendimiento en ecuador. Descargado de http: / / www . uasb. edu .ec/

Contreras, E. (2004). Evaluación social de inversiones públicas: enfoques alternativos y su aplicabilidad para latinoamérica [Manual de software informático].

de Colombia, C. (26 de enero de 2006). Ley 1014 de fomento a la cultura del emprendimiento. En Diario oficial no. 46.164.

de Comercio de Chile, I. N. (2013). Curso de gestión comercial para empresarios, auspiciado y publicado por la cámara de chile.

de la Comunidad Europea, C. (2011). Manual gestión del ciclo de un proyecto, enfoque integrado y marco lógico [Publicación Seriada: Serie métodos e instrumentos para la gestión del ciclo de un proyecto].

de Santa Elena, G. A. D. P. (2015). Actualización del programa de desarrollo y ordenamiento territorial de santa elena.

Duarte, S., y E., J. (2013). Emprendedor: Hacia un emprendimiento sostenible. Editorial Alfaomega. Colombia.

Fonseca, P. (2014). El producto interno bruto. análisis económico. Descargado de http://www. analisiseconomico.info/ index.php/opinion2/414

Francisco, A. (2004). El enfoque del desarrollo económico local. cuaderno de capacitación no. 1 [Manual de software informático].

GEM-Ecuador. (2014). Espae-espol.

González, S. (2010). De la ciudad a la metrópoli. una interpretación teórica del fenómeno expansivo ligado a la vivienda a la vulnerabilidad y a la pobreza: El caso del área metropolitana de monterrey, nuevo león, méxico. Revista INVI, 25, 69. Descargado de http://http://www.scielo .cl/scielo.php?pid=S0718

Group, S. (2013). Los resultados de la administración. Descargado de https:// www. storebrand.no/site/stb.nsf/Get/ get 735c032930150ee419f1545938d13c3e/ \$EILE/selskapene_i_konsernet_ENG . pdf

Guimarães, R. (1998). La ética de la sustentabilidad y la formulación de políticas de desarrollo. (2), 5-24. 
INEC. (2013). Compendio estadístico 2013. Descargado de http: / / www. ecuadorencifras.gob.ec/

INEC. (2014). Nuevo marco conceptual para la clasificación del empleo en ecuador. Descargado de http: / / ww . ecuadorencifras.gob.ec/

Julca Meza, E. (2011). Evaluación de factores básicos de competencia de emprendimiento empresarial en los estudiantes de turismo: caso de la universidad san martín de porres, lima, perú (Tesis para optar por el grado de Doctora en Ciencias empresariales,).

Kaplan, B. (1993). Social change in the capitalist world.

Lacio, V. e. a. (2014). Global entrepreneusir monitor (gem). espae. ecuador.

Lasio V., O. X. e. I. E., Caicedo G. (2015). Informe de la espae a gem [informe].

Licht, A. N. y. S., y I., J. (2006). The social dimensions of entrepreneurship. En B. Y. M Casson y A. B. y N. Wadeson (Eds.), (p. 514-539). The Oxford handbook of entrepreneurship . New York: Oxford. University Press.

López, J. (2009). Modelos predictivos en actitudes emprendedoras: análisis comparativo de las condiciones de ejecución de las redes bayesianas y la regresión logística (Tesis Doctoral, Facultad de Psicología, Universidad de Almería.) Descargado de http: / / hdl. handle.net/10835/356.

Meadows, D. H., Randers, J. y. M., y L., D. (2006). Los límites del crecimiento 30 a nos después. Barcelona: Galaxia Gutenberg.

Moore, M. (2013). Globalization and social change. New York: Elseiver.

Mundial, B. (2015). Ecuador panorama general. Descargado de http: / / www. bancomundial.org/es / country/ecuador/overview.

OIT. (2007). Conferencia internacional del trabajo. 96 a reunión.

Orrego, C. I. (2008, Jul-Dic de 2008). La dimensión humana del emprendimiento. Revista Ciencias Estratégicas., 16(20), 225-235. Descargado de https://revistas.upb.edu.co/index .php/cienciasestrategicas

Peredo, A. M. y. C., y J., J. (2006). Toward a theory of community based enterprise. Academy of Management Review, 31, 309-328.

Pereira, L. F. (2007). La evolución del espíritu empresarial, como campo del conocimiento. hacia una visión sistémica y humanista. Cuadernos de Administración., 20(34), 11-37.

Política del estado del bienestar. (s.f.).

Reyes, G. (2009, Primer Semestre 2009). Teorías de desarrollo económico y social: Articulación con el planteamiento de desarrollo humano. tendencias. Revista de la Facultad de Ciencias Económicas y Administrativas. Universidad de Nari no. Colombia, 10.

Ricardo, D. (1817). Principios de economía política y tributación. En D. F. México (Ed.), Tomo $i$ : Obras y correspondencia. Fondo de Cultura Económica.

Shapero, A. (1981). Entrepreneurship. key to self-renewing economies. Economic Development Commentary, 5, 19-23.

Shapero, L., A. y Sokol. (1982). "the social dimensions of entrepreneurship". En The encyclopedia of entrepreneurship (p. 72-90). Englewood Cliffs . PrenticeHall.

Smith, A. . (1910). Indagación acerca de la naturaleza y causas de la riqueza de las naciones (Vol. 1). Londres.

Yunta., C. L. (2010). Autogestión y solidaridad en la construcción de prácticas económicas alternativas en la ciudad de buenos aires. Experiencias de autogestión en el seno de los Movimientos Sociales. Balance y Perspectivas. Descargado de http://.amazona.com/academia .edu.documents//autogestion.pdf

Recibido: 24 de mayo de 2017

Aceptado: 17 de julio de 2017

Valencia Cruzaty, Lilia: Ingeniera Comercial graduada de la Universidad de Guayaquil en el año 2003. Es Magister en Administración de Empresas, mención en Recursos Humanos y Marketing de la Universidad de Guayaquil desde el año 2011, y Magister en Gerencia Educativa de la Universidad Estatal de Bolívar desde el año 2012. Actualmente se encuentra cursando su Doctorado en Administración en la Universidad Nacional Mayor de San Marcos, Lima Perú. 